\title{
BOOLEAN REDUCTS OF RELATION AND CYLINDRIC ALGEBRAS AND THE CUBE PROBLEM
}

\author{
H. ANDREKA
}

\begin{abstract}
It is shown that not every Boolean algebra is the Boolean part of a nondiscrete relation or cylindric algebra, but every nonatomless Boolean algebra is. Solutions of Tarski's Cube Problem for nondiscrete relation and cylindric algebras are given.
\end{abstract}

Introduction. Relation algebras (RAs) and cylindric algebras (CAs) are Boolean algebras (BAs) endowed with additional structure. The question naturally arises: Which BAs are Boolean parts (i.e. Boolean reducts) of RAs or CAs?

This question can be considered as a representation problem for BAs, too: Which BA can be represented as a BA of some binary relations on a set $U$ closed under composition, inversion, and containing the identity relation on $U$ (this is the RA case); and which BA can be represented as a BA of $n$-ary relations closed under, roughly, first-order definability, i.e. containing with every relation $R_{1}, \ldots, R_{k}$ the relations first-order definable (with $n$ variables) from them, too?

$\mathrm{RA}$ and $\mathrm{CA}_{\alpha}$ denote the classes of RAs and CAs of dimension $\alpha$ respectively. There is a trivial way of turning every BA into a RA or a CA $\mathrm{A}_{\alpha}$; these RAs and CAs are called "Boolean" and "discrete" ones (cf. [7, p. 276 and 5, 1.3.11]). By [5, 1.2.14], every BA is the Boolean part of a nondiscrete $\mathrm{CA}_{1}$. J. D. Monk in 1981 and independently R. Laver in 1984 asked the question: Which BAs are Boolean parts (reducts) of nondiscrete $\mathrm{CA}_{\alpha} \mathrm{s}, \alpha \geqslant 2$ ? Theorems 1 and 2 give partial answers.

To $\mathrm{CA}_{\alpha} \mathrm{s}$ there correspond another BA, the so-called zero-dimensional part $3 \mathrm{~d} \mathfrak{A}$ of a $\mathrm{CA}_{\alpha} \mathfrak{A}$. By $[5,2.4 .35]$, every $\mathrm{BA}$ is the zero-dimensional part of a hereditarily nondiscrete $\mathrm{CA}_{\alpha}$ (for all $\alpha>0$ ). In the last part of the paper we use this fact (together with Ketonen's solution for BAs) to give a full solution of Tarski's Cube Problem for $\mathrm{CA}_{\alpha} \mathrm{s}$ as well as for RAs (of course we mean nondiscrete $\mathrm{CA}_{\alpha} \mathrm{s}$ and RAs here). See Theorems 3 and 4. This solves that part of Problem 2.4 of [5] which was still left open in [6, p. 127].

The definitions of RA and $\mathrm{CA}_{\alpha}$, both originating with Tarski, can be found e.g. in $[5,7,8,10]$ and in $[5,6]$ respectively. Below we recall that part of their definitions that will be needed in the present paper.

Received by the editors April 2, 1985 and, in revised form, August 19, 1985 and February 11, 1986. 1980 Mathematics Subject Classification (1985 Revision). Primary 03G05, 03G25; Secondary 03E15, $06 \mathrm{E} 99$. 
LeMma 2. There is a $B A \mathfrak{B}$ such that for any two disjoint nonzero elements $\underline{a}, \underline{b}$ of $\mathfrak{B}$ there is a $\kappa$ such that $\underline{a}$ has a $\kappa$-partition while $\underline{b}$ has no $\kappa$-partition.

Proof. We use the BA-construction (tree algebras) of [1,2]. Let $T$ be a tree satisfying (1) the height of $T$ is $\omega$ and $T$ has a single root and (2) $(\forall u, t \in T$ ) $\left[u \neq t \Rightarrow\left|A_{u}\right| \neq\left|A_{t}\right|\right.$ and $\left|A_{u}\right|$ is regular and uncountable], where $A_{u}$ is the set of immediate successors to $u$ in $T$. Then the BA $B_{T}$ generated by the principal filters of $T$ satisfies the requirements for $B$ in Lemma 2 . This can be proved the same way as Claim 1 is proved in the proof of Theorem 2.4 of [1] , but using a relativized version of Lemma 2.2 of [1]. Q.E.D.

We are ready to prove Theorem 1 . Let $\mathfrak{B} \in \mathrm{BA}$ be as stated in Lemma 2 . Let $\mathfrak{U} \in \mathrm{CA}_{\alpha}, \alpha \geqslant 2$, be nondiscrete. Then $D \stackrel{d}{=} d_{01} \cdot c_{1}-d_{01} \neq 0,-d_{01} \neq 0$, and clearly $D \cdot-d_{01}=0$. By Lemma 1 then $\mathfrak{B}$ is not the Boolean reduct of $\mathfrak{A}$. By Theorem 5.3.17 and Definition 5.3.7 of [5], for every nondiscrete $\Re \in$ RA there is a nondiscrete $\mathbb{E} \in \mathrm{CA}_{2}$ such that the Boolean reducts of $\Re$ and $\mathbb{E}$ coincide. Therefore $\mathfrak{B}$ is not the Boolean reduct of any nondiscrete RA either. Q.E.D. (Theorem 1)

We note that one can easily state and prove Lemma 1 by writing $\mathfrak{A} \in \mathrm{RA}$, $D=1^{\prime} \cdot\left[\left(-1^{\prime}\right) ; 1\right],-1^{\prime}$, and $b_{i} \stackrel{d}{=}\left(a_{i} ; 1\right)-1^{\prime}$ instead of $\mathfrak{U} \in \mathrm{CA}, D=d_{01} \cdot c_{1}-d_{01}$, $-d_{01}$, and $b_{i} \stackrel{d}{=} c_{1} a_{i}-d_{01}$, respectively, this way obtaining a direct proof for RAs.

Proof of Theorem 2(a). Let $\mathfrak{B} \in \mathrm{BA}$ and $a \in A t \mathfrak{B}$. For all $x, y \in B$ define $x \stackrel{d}{=} x, 1^{\prime} \stackrel{d}{=} a$, and

$$
x ; y \stackrel{d}{=} \begin{cases}1^{\mathfrak{H}} & \text { if } 0^{\mathfrak{B}}, a \notin\{x, y\}, \text { and } x \cdot y \neq 0, \\ 1^{\mathfrak{B}}-a & \text { if } 0^{\mathfrak{B}}, a \notin\{x, y\}, \text { and } x \cdot y=0, \\ 0^{\mathfrak{B}} & \text { if } 0^{\mathfrak{B}} \in\{x, y\}, \\ x & \text { if } a=y, \\ y & \text { if } a=x .\end{cases}
$$

Let $\Re$ be the algebra we obtain from $\mathfrak{B}$ by endowing it with the above operations, i.e. $\Re=\left\langle\mathfrak{B}, ;, \cup, 1^{\prime}\right\rangle$. Now it is not difficult to check that $\Re$ is an integral RA. That $\Re$ is isomorphic to a relation set algebra follows from results in [11]. We may assume that $\mathfrak{B}$ is a Boolean set algebra with base $U$ and that $a$ is a one-element set. The algebra $\mathscr{E}=\mathscr{E}_{|U|}(\{1,2,3\})$ is defined in Definition 2.4 of [11] and shown to be an RA in [11, Theorem 2.5(5)]. That $\mathscr{E}$ is an Rs follows from Theorem 5.19 of [11] since it is not difficult to check that $\mathscr{E}$ satisfies condition 5.13(x). Finally, $\Re$ is in Rs because it is a subalgebra of $\mathscr{E}$. Q.E.D. (Theorem 2(a))

Proof of Theorem 2(b). Let $n \in \omega$. Assume that $\mathfrak{B} \in \mathrm{BA}$ has at least $\pi n-1$ atoms. Let $P \stackrel{d}{=}\{E: E$ is a partition of $n$ such that not every block of $E$ is one-element $\}$. Then $|P|=\pi n-1$. Let $b: P \mapsto A t \mathfrak{B}$ be a one-one mapping. We shall write $b_{E}$ instead of $b(E)$.

Case 1. $\Sigma(\operatorname{Rg} b)=1$ in $\mathscr{B}$. Define $f\left(b_{E}\right) \stackrel{d}{=}\left\{s \in{ }^{n}(n-1): \operatorname{ker}(s)=E\right\}$ for $E \in P$ and $f(a) \stackrel{d}{=} \cup\left\{f\left(b_{E}\right): b_{E} \leqslant a\right\}$ for $a \in B$. Then $f$ is an isomorphism of $\mathfrak{B}$ onto the Boolean part of the smallest $\mathrm{Cs}_{n}$ with base $n-1$ (cf. e.g. [5, 2.4.69]). 
Case 2. $\Sigma(\operatorname{Rg} b) \neq 1$ in $\mathfrak{B}$. We may assume that $\mathfrak{B}$ is a Boolean set algebra with base $U$. Let $K \stackrel{d}{=} U \sim \bigcup\left\{b_{E}: E \in P\right\}$. Then $K \neq 0$. We may assume that $|K| \geqslant \omega$. (There is a construction for finite $K$, too, but we want to avoid case-distinction.) We will construct $\left\langle H_{\kappa}: \kappa \in K\right\rangle$ with the following properties. Let $\bar{D} \stackrel{d}{=}\left\{s \in{ }^{n} K: s\right.$ is one-one $\}$.

(i) $\bigcup\left\{H_{\kappa}: \kappa \in K\right\}=\bar{D}$.

(ii) $H_{\kappa} \cap H_{\delta}=0$ if $\kappa, \delta \in K, \kappa \neq \delta$.

(iii) $C_{i} H_{\kappa}=C_{i} \bar{D}$ for each $\kappa \in K$ and $i \in n$.

Let $\langle K,+,-\rangle$ be any commutative group on $K$. Let $\left\langle K_{\kappa}: \kappa \in K\right\rangle$ be such that $\bigcup\left\{K_{\kappa}: \kappa \in K\right\}=K,(\forall \kappa \in K)\left|K_{\kappa}\right| \geqslant n$, and $(\forall \kappa, \delta \in K)\left[\kappa \neq \delta \Rightarrow K_{\kappa} \cap K_{\delta}=0\right]$. Define

$$
H_{\kappa} \stackrel{d}{=}\left\{s \in \bar{D}: \sum\left\{s_{i}: i \in n\right\} \in K_{\kappa}\right\}, \quad \text { for every } \kappa \in K .
$$

Now $\left\langle H_{\alpha}: \kappa \in K>\right.$ clearly satisfies (i)-(ii). Let $\kappa \in K, i \in n$, and $s \in \bar{D}$ be fixed. Let $L \stackrel{d}{=}\left\{s_{j}: j \in n, j \neq i\right\}, \sigma \stackrel{d}{=} \sum L$, and $S \stackrel{d}{=}\left\{\delta-\sigma: \delta \in K_{\kappa}\right\}$. Then $|S| \geqslant n$, hence $S \sim L \neq 0$. Let $u \in S \sim L$. Let $z \in{ }^{n} K$ be such that $z_{i}=u$ and $(\forall j \in n, j \neq i) z_{j}$ $=s_{j}$. Then $z \in \bar{D}$ by $u \notin L$ and $\sum\left\{z_{j}: j \in n\right\}=\sigma+u \in K_{\kappa}$ by $u \in S$. Thus $z \in H_{\kappa}$, showing $C_{i} \bar{D}=C_{i} H_{\kappa}$. We have seen that (iii) holds for $\left\langle H_{\kappa}: \kappa \in K\right\rangle$. Define

$$
f\left(b_{E}\right) \stackrel{d}{=}\left\{s \in{ }^{n} K: \operatorname{ker}(s)=E\right\} \quad \text { for } E \in P
$$

and

$$
f(a) \stackrel{d}{=} \cup\left\{H_{\kappa}: \kappa \in a \cap K\right\} \cup \bigcup\left\{f\left(b_{E}\right): b_{E} \leqslant a, E \in P\right\} \quad \text { for } a \in B .
$$

Let $\mathbb{C}$ be the full $\mathrm{Cs}_{n}$ with base $K$. Then $f$ is an embedding of $\mathfrak{B}$ into the Boolean part $\mathfrak{B} \mathfrak{l} \mathbb{E}$ of $\mathbb{C}$ by ${ }^{n} K \sim \bar{D}=\bigcup\left\{f\left(b_{E}\right): E \in P\right\}, f\left(1^{\mathfrak{B}}\right)={ }^{n} K$, and by (i)-(ii). Let $F \stackrel{d}{=}\{f(a): a \in B\}$. We will show that $F$ is closed under the operations of $\mathbb{E}$. Let $i<j<n$. Then $d_{i j}^{\sqrt{5}}=f\left(b_{E}\right)$ where $E$ is the partition of $n$ keeping $i, j$ together and leaving all the other points separated. Let $\mathfrak{M}$ be the smallest subalgebra of $\mathfrak{E}$. Then $M=\operatorname{Sg}^{\left({ }^{(\mathscr{C})}\right)}\left\{d_{i j}^{\Subset}: i<j<n\right\}$ by $[5,2.2 .24]$. Hence $M \subseteq F$. Let $a \in B$ be arbitrary. Let $i \in n$. If $a \cap K=0$ then $a=\sum\left\{b_{E}: b_{E} \leqslant a, E \in P\right\}$, hence $f(a) \in M$, therefore $c_{i} f(a) \in M \subseteq F$. Assume $a \cap K \neq 0$. Then $c_{i} f(a)=c_{i} \bar{D}+\sum\left\{c_{i} f\left(b_{E}\right): b_{E} \leqslant\right.$ $a, E \in P\} \in M \subseteq F$ by (iii). We have seen that $F$ is closed under $c_{i}, d_{i j}(i, j \in n)$. Q.E.D. (Theorem 2(b))

REMARK. The proof of Theorem 2(b) goes through for $\mathrm{Cs}_{\alpha}$ with $\alpha \geqslant \omega$. Then we obtain the following: If $\mathfrak{B} \in \mathrm{BA}$ is such that $(\exists H \subseteq A t \mathfrak{B})[|H|=|\alpha|$ and $(\forall L \subseteq$ $H) \sup L$ exists in $\mathscr{B}$ ], then $\mathscr{B}$ is the Boolean part of an integral $\mathrm{Cs}_{\alpha}$. The only change in the proof is that we use a Ws $s_{\alpha}$ instead of a $\mathrm{Cs}_{\alpha}$ in order to be able to speak about $\sum \operatorname{Rg}(s)$ for $s \in 1^{\mathbb{E}}$.

In the proofs of Theorems 3 and 4 we will use the notation of [5] without introducing it. 
If $U$ is a set, then $P U$ denotes its powerset. The full relation set algebra with base $U$ is the algebra

$$
\left\langle P(U \times U), \cup, \cap, \sim, U \times U, \varnothing, \mid,,^{-1}, \mathrm{Id}_{U}\right\rangle,
$$

where $R \mid S$ denotes the composition of the relations $R$ and $S, R^{-1}$ is the inverse of the relation $R$, and $\operatorname{Id}_{U}$ denotes the identity relation $\{(u, u): u \in U\}$ on $U$. In RA theory, the operations composition, inversion, and identity are denoted by ; , $u$, and $1^{\prime}$ respectively. A relation set algebra (Rs) is a subalgebra of a full one. RA is a class of algebras defined with finitely many equations such that Rs $\subseteq \mathrm{RA}$.

Let $\alpha$ be an ordinal. The full cylindric set algebra of dimension $\alpha$ with base $U$ is the algebra

$$
\left\langle P\left({ }^{\alpha} U\right), \cup, \cap, \sim,{ }^{\alpha} U, \varnothing, C_{i}, D_{i j}: i, j \in \alpha\right\rangle,
$$

where if $X \subseteq{ }^{\alpha} U$ and $i, j \in \alpha$ then

$$
C_{i} X \stackrel{d}{=}\left\{s \in{ }^{\alpha} U:(\exists z \in X)(\forall j \in \alpha, j \neq i) z_{j}=s_{j}\right\}
$$

and

$$
D_{i j} \stackrel{d}{=}\left\{s \in{ }^{\alpha} U: s_{i}=s_{j}\right\}
$$

The unary operations $C_{i}$ are called cylindrifications and correspond to quantification " $\exists v_{i}$," and the constants $D_{i j}$ are called the diagonals and correspond to the equality " $v_{i}=v_{j}$." A cylindric set algebra of dimension $\alpha\left(\mathrm{Cs}_{\alpha}\right)$ is a subalgebra of a full one. Thus the universe of a $\mathrm{Cs}_{\alpha}$ consists of $\alpha$-ary relations and is closed under first-order (with $\alpha$ variables) definability. $\mathrm{CA}_{\alpha}$ is a class of algebras defined by finitely many (if $\alpha$ is finite) equations such that $\mathrm{Cs}_{\alpha} \subseteq \mathrm{CA}_{\alpha}$.

In $\S 1$ we formulate the results and in $\$ 2$ we give the proofs.

ACKNOWLEDGEMENTS. Theorems 1 and 2 of this paper were obtained in the stimulating atmosphere of the Boolean Algebra Meeting in Oberwolfach, Germany, January 1985. We want to express our thanks to G. Brenner, H. Dobbertin, and J. D. Monk for many stimulating discussions.

1. Formulating the results. We call an RA nondiscrete iff $1^{\prime} \neq 1$ in it. Recall from [5] that a $\mathrm{CA}_{\alpha}$ is nondiscrete iff $d_{01} \neq 1$ in it.

THEOREM 1. There is a BA which is not the Boolean part of any nondiscrete $C A_{\alpha}$, $\alpha \geqslant 2$; hence, it is also not the Boolean part of any nondiscrete $R A$.

A relation algebra is called integral if $1^{\prime}$ is an atom in it. Integral RAs have been extensively investigated, e.g. in $[3,7,8,10-12]$. We call a $\mathrm{CA}_{\alpha} \mathfrak{A}$ integral iff $\left|\mathrm{Nr}_{1} \mathfrak{A}\right| \leqslant 2$. (This expresses the fact that no proper subset of the base of $\mathfrak{A}$ is definable.) This is equivalent to saying that $d_{01}$ is an atom in $\mathrm{Nr}_{2} \mathfrak{A}$, or if $\alpha<\omega$ then to saying that $\Pi\left\{d_{i j}: i<j<\alpha\right\}$ is an atom in $\mathfrak{A}$, or to saying that $\Re \mathfrak{I} \mathfrak{U}$ is integral if $\alpha \geqslant 3$. Note that the only discrete integral $\mathrm{CA}_{\alpha}$ is the two-element one.

THEOREM 2. (a) Let $\mathfrak{B} \in B A$ have at least one atom. Then $\mathfrak{B}$ is isomorphic to the Boolean reduct of an integral $\mathrm{Rs}$ (hence of $a \mathrm{Cs}_{2}$, too).

(b) Let $n \in \omega$ and let $\pi n$ denote the number of partitions of $n$. Let $\mathfrak{B} \in B A$ have at least $\pi n-1$ atoms. Then $\mathfrak{B}$ is isomorphic to the Boolean reduct of some integral $C s_{n}$. 
REMARK. Theorem 2(a) gives a complete characterization of the Boolean parts of each of the following classes: integral RAs, integral Rss, integral $\mathrm{CA}_{2} \mathrm{~s}$, integral $\mathrm{Cs}_{2}$ s. In each case, the class of Boolean reducts is the class of all nonatomless BAs. The hypothesis of Theorem 2(b) is far from being necessary.

We note that for finite BAs the picture is complete: Let $B \in B A$ be finite with $\kappa$ atoms, and let $n \in \omega$. Then $B$ is the Boolean part of a hereditarily nondiscrete $\mathrm{CA}_{n}$ $\left(\mathrm{Cs}_{n}\right.$, integral $\left.\mathrm{Cs}_{n}\right)$ iff $\left[\kappa \in\left\{\pi_{\gamma} n: \gamma \in n\right\}\right.$ or $\left.\kappa \geqslant \pi n\right]$, where $\pi_{\gamma} n$ denotes the number of partitions of $n$ into at most $\gamma$ classes. (This follows from [5, 2.4.69] and Theorem 2(b) herein.) Also, $B$ is the Boolean part of a nondiscrete $\mathrm{CA}_{n}\left(\mathrm{Gs}_{n}\right)$ iff $\kappa \geqslant 2^{(n-1)}$ (this can be proved from [5, 2.4.72]). Further, every finite BA is the Boolean reduct of an integral Rs by Theorem 2(a); compare [10].

THEOREM 3. Let $\alpha$ be any ordinal. Then there is a $C A_{\alpha}$ A with cardinality $|\alpha \cup \omega|$ such that $\mathfrak{A} \cong \mathfrak{A} \times \mathfrak{U} \times \mathfrak{A}$ holds, $\mathfrak{A} \cong \mathfrak{A} \times \mathfrak{U}$ fails, and, in addition, the following hold.

(i) $\mathfrak{A}$ is hereditarily nondiscrete if $\alpha>0$.

(ii) $\mathfrak{A} \in G s_{\alpha}^{\text {reg }} \cap L f_{\alpha}$ if $\alpha>1$.

(iii) $\mathfrak{A} \in I C s_{\alpha} \cap L f_{\alpha}$ if $\alpha \geqslant \omega$.

THEOREM 4. There is a denumerable nondiscrete $R A \Re$ such that $\Re \cong \Re \times \Re \times \Re$ but $\Re \nRightarrow \Re \times \Re$.

\section{Proofs.}

Proof OF TheOREM 1. We establish a property of Boolean reducts of nondiscrete CAs and then we construct a BA that does not have that property.

Definition. An element of a BA has a $\kappa$-partition iff it is the supremum of an antichain of size $\kappa$. More formally: Let $x \in \mathfrak{B} \in \mathrm{BA}$ and let $\kappa$ be an ordinal. Then $\left\langle a_{i}: i \in \kappa\right\rangle \in{ }^{\kappa} B$ is a $\kappa$-partition of $x$ iff $(\forall i<j<\kappa)\left(a_{i} \neq 0=a_{i} \cdot a_{j}\right.$ and $x=$ $\left.\sum\left\{a_{i}: i \in \kappa\right\}\right)$.

Lemma 1. Let $\mathfrak{U} \in C A_{\alpha}, \alpha \geqslant 2$. If $D \stackrel{d}{=} d_{01} \cdot c_{1}\left(-d_{01}\right)$ has a $\kappa$-partition, then $-d_{01}$ has a $\kappa$-partition, too.

Proof. Let $\left\langle a_{i}: i \in \kappa\right\rangle$ be a $\kappa$-partition of $D$. Define $b_{i} \stackrel{d}{=}\left(c_{1} a_{i}\right)-d_{01}$ for every $i \in \kappa$. We will show that $\left\langle b_{i}: i \in \kappa\right\rangle$ is a $\kappa$-partition of $-d_{01} . b_{i} \neq 0$ because of the following: Assume $b_{i}=c_{1} a_{i}-d_{01}=0$. By $[5,1.5 .5]$ we have $c_{1} a_{i} \cdot d_{01}=a_{i}$ since $a_{i} \leqslant d_{01}$. Now $c_{1} a_{i}=\left(c_{1} a_{i} \cdot d_{01}\right)+\left(c_{1} a_{i}-d_{01}\right)=a_{i}$. But then $-a_{i}=c_{1}-a_{i} \geqslant$ $c_{1}-d_{01} \geqslant a_{i}$, a contradiction. $b_{i} \cdot b_{j}=0$ if $i \neq j$ because of the following:

$$
c_{1} a_{i} \cdot c_{1} a_{j}=c_{1}\left(a_{i} \cdot c_{1} a_{j}\right)=c_{1}\left(a_{i} \cdot d_{01} \cdot c_{1} a_{j}\right)=c_{1}\left(a_{i} \cdot a_{j}\right)=c_{1} 0=0,
$$

hence $b_{i} \cdot b_{j}=\left(c_{1} a_{i}-d_{01}\right) \cdot\left(c_{1} a_{j}-d_{01}\right)=0 . \sum\left\{b_{i}: i \in \kappa\right\}=-d_{01}$ because of the following: By $[5,1.2 .6(\mathrm{i})]$ we have

$$
\sum\left\{c_{1} a_{i}: i \in \kappa\right\}=c_{1} \sum\left\{a_{i}: i \in \kappa\right\}=c_{1}\left(d_{01} \cdot c_{1}-d_{01}\right)=c_{1}-d_{01} .
$$

$\left(\sum\left\{x_{i}: i \in I\right\}\right) \cdot b=\sum\left\{x_{i} \cdot b: i \in I\right\}$ holds in every BA, hence

$$
\sum\left\{c_{1} a_{i}-d_{01}: i \in \kappa\right\}=\left(c_{1}-d_{01}\right)-d_{01}=-d_{01} \text {. }
$$

Q.E.D. (Lemma 1) 
Proof of Theorem 3. Let $\mathfrak{B}$ be a denumerable BA such that $\mathfrak{B} \cong \mathfrak{B} \times \mathfrak{B} \times \mathfrak{B}$ while $\mathfrak{B} \not \mathfrak{B} \times \mathfrak{B}$. Such a BA exists by Ketonen's proof [9]. Let $\mathfrak{C}$ be a minimal $\mathrm{Cs}_{\alpha}$, with infinite base if $\alpha \geqslant \omega$. Then $\mathbb{E}$ is directly indecomposable, $\mathbb{E} \in \mathrm{Cs}_{\alpha}^{\text {reg }}$, and $|C| \leqslant|\alpha \cup \omega|$. Construct the algebra $\mathfrak{Y}^{\prime} \in \mathrm{CA}_{\alpha}$ as in the proof of 2.4.35 of [5]. Then $\mathfrak{U}^{\prime} \subseteq{ }^{U} \mathbb{C}$ for some $U$ by [5, statement (4), p. 316], hence $\mathfrak{A}^{\prime} \in I \mathrm{Gs}_{\alpha}^{\text {reg }}$ and further $\mathfrak{H}^{\prime} \in I \mathrm{Cs}_{\alpha}$ if $\alpha \geqslant \omega$ by $S P_{\infty} \mathrm{Cs}_{\alpha} \subseteq I_{\infty} \mathrm{Cs}_{\alpha}$ for $\alpha \geqslant \omega$ (cf. [5, 3.1.106]). $\mathfrak{A}^{\prime} \in L f_{\alpha}$ by [5, statement (5), p. 316]. $\mathfrak{U}^{\prime}$ is hereditarily nondiscrete for $\alpha>0$ by (6) on p. 316 of [5]. Also, $\left|A^{\prime}\right|=|\alpha \cup \omega|$ because $|C| \leqslant|\alpha \cup \omega|,|B|=\omega$ and since $A^{\prime} \subseteq\{f \in$ ${ }^{U} C:\left\{f^{-1 \star} c: c \in C\right\}$ is a finite subset of $\left.B\right\}$ by the definition of $A^{\prime}$. It remains to show that $\mathfrak{U}^{\prime} \cong \mathfrak{U}^{\prime} \times \mathfrak{U}^{\prime} \times \mathfrak{U}^{\prime}$ and $\mathfrak{U}^{\prime} \not \mathfrak{U}^{\prime} \times \mathfrak{U}^{\prime}$. By $\mathfrak{B} \cong \mathfrak{B} \times \mathfrak{B} \times \mathfrak{B}$ and by [5, 2.4.7], there are $b_{0}, b_{1}, b_{2} \in B$ such that

$$
\begin{aligned}
& \mathfrak{R} \mathfrak{I}_{1} \mathfrak{B}=\mathfrak{B} \cong \mathfrak{A I}_{b_{i}} \mathfrak{B} \quad \text { for } i \in 3 \text {, } \\
& \sum\left\{b_{i}: i \in 3\right\}=1 \text { and } b_{i} \cdot b_{j}=0 \text { for } i<j<3 .
\end{aligned}
$$

The desired decomposition of $\mathfrak{U}^{\prime}$ now follows from [5, 2.4.35(ii)]. On the other hand, if $\mathfrak{A}^{\prime} \cong \mathfrak{U}^{\prime} \times \mathfrak{A}^{\prime}$, then 2.4.3 and 2.4.35(i) of [5] yield $\mathfrak{B} \cong \mathfrak{B} \times \mathfrak{B}$ contradicting the choice of $\mathfrak{B}$. Q.E.D. (Theorem 3 )

Proof of TheOrEm 4. Let $\mathfrak{A} \in \mathrm{Gs}_{3}$ be such that $\mathfrak{A} \cong \mathfrak{U} \times \mathfrak{U} \times \mathfrak{A}$ but $\mathfrak{B} \not \mathfrak{B} \times$ $\mathfrak{B}$ for $\mathfrak{B} \stackrel{d}{=} \mathfrak{Z} \mathfrak{d}$. Such an $\mathfrak{A}$ exists by inspecting the proof of Theorem 3 . Let $\mathfrak{R} \stackrel{d}{=} \mathfrak{R} \mathfrak{a}$. Then $\mathfrak{R} \in \mathrm{RA}$ by $[\mathbf{5}, 5.3 .9]$. By [5, 5.3.11] we have $\mathfrak{R} a(\mathfrak{U} \times \mathfrak{U} \times \mathfrak{U})=$ $\mathfrak{R} a \mathfrak{U} \times \mathfrak{R} a \mathfrak{A} \times \mathfrak{R} a \mathfrak{A}$, thus $\mathfrak{R} \cong \mathfrak{R} \times \mathfrak{R} \times \mathfrak{R}$. Assume $\mathfrak{R} \cong \mathfrak{R} \times \mathfrak{R}$. Let $\mathfrak{B}$ denote the BA of all ideal elements of $\Re$, i.e. $\mathfrak{B}=\langle$ Ie $\Re,+, \cdot,-, 0,1\rangle$, where Ie $\Re \stackrel{d}{=}\{x \in$ $R: 1 ; x ; 1=x\}$. It can be checked that Ie $\Re=Z \mathrm{~d} \mathfrak{A}$, as follows. Let $x \in R=\mathrm{Nr}_{2} \mathfrak{A}$. Then $1 ; x=c_{2}\left(s_{2}^{1} 1 \cdot s_{2}^{0} x\right)=c_{2} c_{0}\left(d_{02} \cdot c_{2} x\right)=c_{0} c_{2} x=c_{0} x$ and similarly $x ; 1=c_{1} x$. Thus $\mathfrak{P}=\mathfrak{B}$. Clearly, $\operatorname{Ie}(\mathfrak{R} \times \mathfrak{R})=$ Ie $\mathfrak{R} \times$ Ie $\mathfrak{R}$, hence $\mathfrak{R} \cong \mathfrak{R} \times \mathfrak{R}$ implies $\mathfrak{B} \cong \mathfrak{B}$ $\times \mathfrak{B}$, contradicting $\mathfrak{B} \neq \mathfrak{B} \times \mathfrak{B}$. Q.E.D. (Theorem 4)

\section{REFERENCES}

1. G. M. Brenner, A simple construction for rigid and weakly homogeneous Boolean algebras answering a question of Rubin, Proc. Amer. Math. Soc. 87 (1983), 601-606.

2. G. M. Brenner and J. D. Monk, Tree algebras and chains, Universal Algebra and Lattice Theory, Lecture Notes in Math., vol. 1004, Springer-Verlag, New York, 1983, pp. 54-66.

3. S. D. Comer, Combinatorial aspects of relation algebras, Algebra Universalis 18 (1984), 77-94.

4. W. Hanf, On some fundamental problems concerning isomorphism of Boolean algebras, Math. Scand. 5 (1957), 205-217. 1985.

5. L. Henkin, J. D. Monk and A. Tarski, Cylindric algebras. I, II, North-Holland, Amsterdam, 1971,

6. L. Henkin, J. D. Monk, A. Tarski, H. Andréka, I. Németi, Cylindric set algebras, Lecture Notes in Math., vol. 883, Springer-Verlag, Berlin, 1981.

7. B. Jónsson, Varieties of relation algebras, Algebra Universalis 15 (1982), 273-298.

8. B. Jónsson and A. Tarski, Boolean algebras with operators. II, Amer. J. Math. 74 (1952), 127-162.

9. J. Ketonen, The structure of countable Boolean algebras, Ann. of Math. (2) 108 (1978), 41-89.

10. R. Maddux, Finite integral relation algebras, Universal Algebra and Lattice Theory (Proc. Charleston 1984), Lecture Notes in Math., vol. 1149, Springer-Verlag, Berlin, 1985, pp. 175-197.

11. Some varieties containing relation algebras, Trans. Amer. Math. Soc. 272 (1982), 501-526.

12. R. McKenzie, Representations of integral relation algebras, Michigan Math. J. 17 (1970), 279-287.

Mathematical Institute of the Hungarian Academy of Sciences, Budapest, Pf. 127, H-1364 HUNGARY 